\title{
Multi-angle Face Detection Based on DP-Adaboost
}

\author{
Ying-Ying Zheng Jun Yao \\ School of Mechanical and Electronic Engineering and Automation, Shanghai University, Shanghai 200072, China
}

\begin{abstract}
Although important progresses have been already made in face detection, many false faces can be found in detection results and false detection rate is influenced by some factors, such as rotation and tilt of human face, complicated background, illumination, scale, cloak and hairstyle. This paper proposes a new method called DP-Adaboost algorithm to detect multi-angle human face and improve the correct detection rate. An improved Adaboost algorithm with the fusion of frontal face classifier and a profile face classifier is used to detect the multi-angle face. An improved horizontal differential projection algorithm is put forward to remove those non-face images among the preliminary detection results from the improved Adaboost algorithm. Experiment results show that compared with the classical Adaboost algorithm with a frontal face classifier, the textual DP-Adaboost algorithm can reduce false rate significantly and improve hit rate in multi-angle face detection.
\end{abstract}

Keywords: Multi-angle face detection, Adaboost, classifier fusion, improved horizontal differential projection, false face.

\section{Introduction}

Many applications in vision system and image analysis used face detection as an important feature in their system development ${ }^{[1]}$. Face detection has broad application prospects in the applications such as person identification and video surveillance ${ }^{[2,3]}$. Therefore, it is a hot research topic in the field of pattern recognition.

With the rapid development of technology in computer vision, more and more face detection methods were put forward around the world. Turk and Pentland ${ }^{[4]}$ proposed the PCA to detect human face and Yang and Hang ${ }^{[5]}$ discussed a typical example using face detection method based on priori knowledge in 1994. Cootset et al. ${ }^{[6]}$ submitted active shape models (ASM) and active appearance model (AAM), which were two classic methods for deformable template. Support vector machine (SVM) was used to detect human face to classify face and non-face images firstly by Osuna et al. ${ }^{[7]}$ and this research obtained good experiment results. In 2001, Viola and Jones ${ }^{[8]}$ introduced an Adaboost algorithm to the face and non-face detection. It used integral figure and strong classifier to gain a high detection rate in real-time classification. Since then, the Adaboost algorithm has been widely used in many tasks of object detection.

Although good effects have already been achieved in frontal face detection, the research on non-frontal face detection is still far from the application requirement because of the influence from rotation and tilt of human face in an image ${ }^{[9-11]}$. Multi-angle face detection is defined that the human face to be detected rotates a number of different viewing angles. It is a frontal face or a profile face ${ }^{[12]}$. Compared with frontal face detection, the study of multi-angle face detection is very difficult and its practical application is rare. Rowley et al. ${ }^{[13]}$ carried out a research on router neural network to detect human face with a rotation angle by

Regular pape

Manuscript received September 30, 2013; accepted May 5, 2014

Recommended by Guest Editor Xin Sun

(C) Institute of Automation, Chinese Academy of Science and

Springer-Verlag Berlin Heidelberg 2015 changing a rotation face in an image into a frontal face. Li and Zhang ${ }^{[14]}$ took the advantage of FloatBoost algorithm to combine several weak classifiers into strong classifier to generate a face detection system with a pyramid hierarchy to detect many kinds of rotated human face. It was a big step forward in the field of face detection. Schneiderman and Kanade ${ }^{[15]}$ used view-based multiple detectors and statistical modeling with histograms to detect faces with out-of-plane rotation. Although some good progresses have been made when using the aforementioned methods in the literatures to detect multi-angle faces in images, many false faces can still be found in the detection result and the false detection rate is influenced by some factors, such as rotation and tilt of human face, complicated background, illumination, scale, cloak and hairstyle.

A novel multi-angle face detection method called DPAdaboost algorithm is presented in this paper to detect multi-angle face in an image and improve the correct detection rate. The Adaboost algorithm for multi-angle face detection with the fusion of frontal face classifier and profile face classifier is used to detect frontal faces and profile faces. By comparing the ratios of overlapped area in these detected faces, an overlap separation method is used to obtain the preliminary detection results. In order to eliminate the false faces among the preliminary detection results, an improved horizontal differential projection algorithm is used in the textual DP-Adaboost algorithm. Experiments show that DP-Adaboost algorithm has a high correct detection rate and a low false detection rate for multi-angle face detection.

The paper is organized as follows. Section 1 introduces the development of multi-angle face detection and gives the purpose of this paper. An overview of DP-Adaboost algorithm is shown in Section 2 to describe its basic method and procedure. In Section 3, classifier fusion of frontal face 
classifier and profile face classifier based on the principle of classical Adaboost algorithm and the overlap separation method is proposed. Considering that false faces are still exist in the preliminary results, an improved horizontal differential projection algorithm is put forward in Section 4 to remove those false detection results. Experiments are given in Section 5 to show the effectiveness of DP-Adaboost algorithm. Finally, Section 6 summarizes the full paper.

\section{An overview of DP-Adaboost}

As the classical Adaboost algorithm, it is very useful to detect the frontal face. However, for the profile face, the effective detection of classical Adaboost algorithm does not perform very well. Therefore, a classifier fusion of frontal face classifier and profile face classifier is designed in the DP-Adaboost algorithm to detect multi-angle face.

When detecting the human face with Adaboost algorithm, many false faces will be found in the detection results. An improved horizontal differential projection method is presented in the DP-Adaboost algorithm to remove false faces in the detected results. Compared with the classical horizontal differential projection method, there are two advantages of the improved horizontal differential projection method. One advantage is that the location of two eyes can be highlighted and the value of peak in the horizontal differential projection image is increased by the weighted method. The other one is that the false detection caused by the problem which is that two eyes are not on the horizontal straight line. And this problem is solved by the improved method. The flow chart of the textual DP-Adaboost algorithm is shown in Fig. 1.

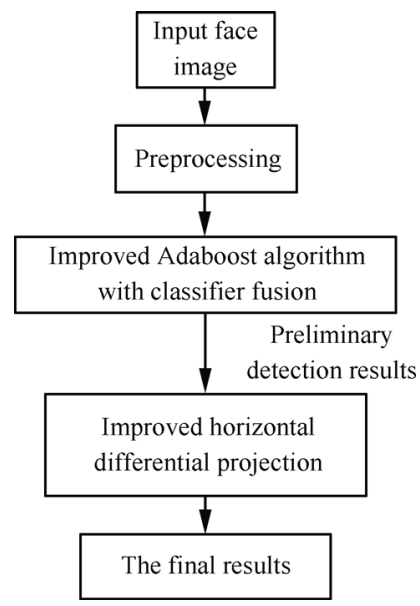

Fig. 1 The flow chart of DP-Adaboost algorithm

In Fig. 1, images are preprocessed firstly with histogram equalization and Gaussian smoothing method to enhance image contrast and weaken the influence of noise.

Then preliminary detection results are obtained by the improved Adaboost algorithm, in which a classifier fusion that combines the frontal face cascade classifier with the profile face cascade classifier is generated based on the strong classifier from Adaboost algorithm. The detailed process of the improved Adaboost algorithm with classifier fusion is shown in Fig. 2. According to the principle of the Adaboost algorithm, a frontal cascade classifier and a profile cascade classifier are obtained through these training frontal face samples, profile face samples and non-face samples. After that, two classifiers are combined to obtain classifier fusion result.

Finally, an improved horizontal differential projection method is applied to the preliminary detection results to eliminate those false detection results and increase the detection rate of the final results.

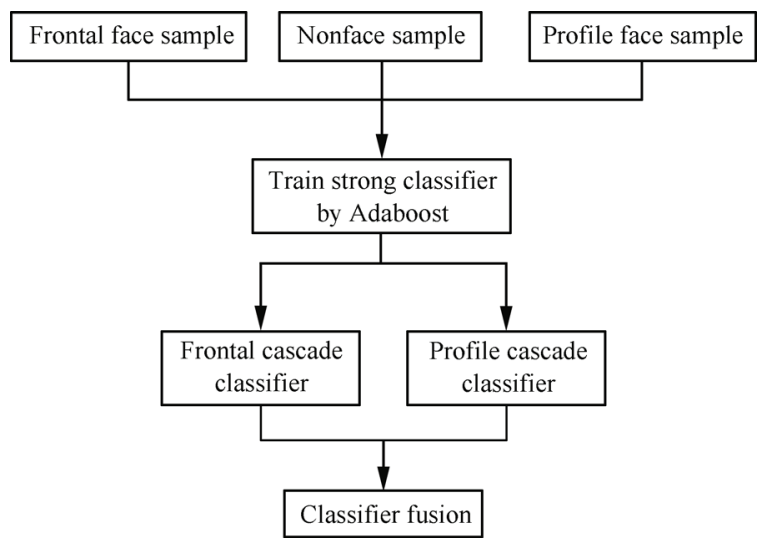

Fig. 2 Improved Adaboost algorithm with classifier fusion

\section{Classifier fusion of Adaboost algo- rithm}

The classical Adaboost algorithm for frontal face detection trains a weak classifier with its corresponding weights for different training sets and then combines a large number of weak classifiers with Haar features into a strong classifier. Finally, a cascade classifier is generated by a series of strong classifiers. For the issue of multi-angle face detection, it is necessary to design frontal face and profile face cascade classifiers and combine them to get detection results.

\subsection{Optimizing strong classifier}

Firstly, weak classifier is trained by 14 kinds of Haar features $^{[16]}$ which includes edge features, line features and center-surround features. These Haar features are shown in Fig. 3. These Haar features encode differences in average intensities between two rectangular regions and they can be calculated rapidly through integral images. The complete Haar feature set is large and contains a mass of redundant information $^{[17]}$. The value of Haar feature is the difference between the sums of the pixels values within the black and white rectangle regions ${ }^{[18]}$. This paper uses the Massachusetts Institute of Technology (MIT) face library and face recognition technology (FERET) face library including face and non-face images as training samples. The size of these images is resized to $20 \times 20$ and the quantity of their Haar features is 80000 . In the literature, there may be three basic types: 


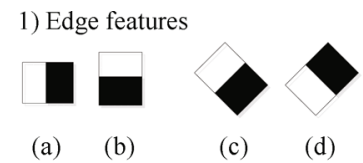

2) Line features
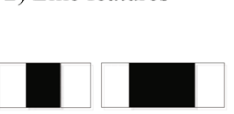

(a)

(b)

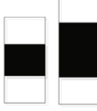

(c) (d)

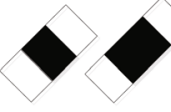

(e) (f)

3) Center-surround fearures

(a) (b)

Fig. 3 Haar features

Integral image is used in Adaboost algorithm to calculate Haar features rapidly. When calculating the sum of grey values of these pixels in specific characteristics of the rectangular, integral image can perform simple arithmetic operations by integral characteristic values of the existing integral points. Because of avoiding repeated calculation of the same integral pixel for different dimensions of the characteristic rectangle, integral image can improve the efficiency of detection ${ }^{[19]}$. The value of integral image for point $(i, j)$ is defined as

$$
i i(i, j)=\sum_{k \leqslant i, l \leqslant j} f(k, l)
$$

where $f(k, l)$ means the pixel value at point $(k, l)$.

For example, these values of integral image in Fig. 4 are defined as $i i_{n}$, where the number point $n$ is from 1 to 6 . The Haar-like edge character of $A$ and $B$ regions can be defined as Sum $_{\text {region by }}$

$$
\left\{\begin{array}{l}
S u m_{A}=i i_{5}+i i_{1}-i i_{2}-i i_{4} \\
\operatorname{Sum}_{B}=i i_{6}+i i_{2}-i i_{5}-i i_{3} \\
\operatorname{Sum}_{\text {region }}=S u m_{A}-S u m_{B} .
\end{array}\right.
$$

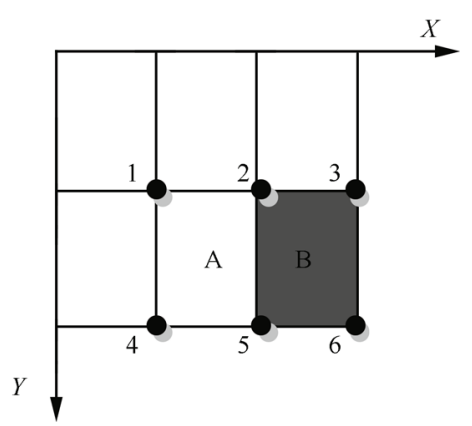

Fig. 4 An example of integral image

Therefore, the integral image contributes to detecting multi-angle face rapidly and optimizing Adaboost algorithm because of abundant Haar features. The algorithm describes as follows ${ }^{[20]}$ :

Step 1. Given a series of training sample $\left(x_{i}, y_{i}\right) . y_{i}$ equaling to 0 means a negative sample and $y_{i}$ equaling to 1 means positive sample. The number of the training samples is $N$.
Step 2. The number of positive samples and negative samples are respectively set as $m$ and $h$. For the positive samples, $w_{i}$ equals to $\frac{1}{2 m}$ and for the negative samples, $w_{i}$ equals to $\frac{1}{2 h}$.

Step 3. For $T$-round training:

1) Normalize the sample weight

$$
W_{t, i}=\frac{W_{t, i}}{\sum_{j=1}^{n} W_{t, j}}
$$

where $t=1,2, \cdots, T$.

2) Training the weak classifier $h(x, f, p, \theta)$ in (4) to determine the optimal threshold of the Haar feature $f$ which makes the minimum classification error $\varepsilon$ in (5) for all training samples

$$
\begin{aligned}
& h(x, f, p, \theta)= \begin{cases}1, & p f(x)<p \theta \\
0, & \text { otherwise }\end{cases} \\
& \varepsilon=\sum_{i} W_{i}\left|h\left(x_{i}\right)-y_{i}\right| .
\end{aligned}
$$

In (4), $f$ is the Haar feature, $\theta$ is threshold, $x$ is the child window for detection, and $p$ is the direction of inequality sign.

3) Update the sample weight $W_{t+1, i}$ :

$$
\begin{aligned}
& W_{t+1, i}=W_{t, i} \beta_{t}^{1-\varepsilon_{t}} \\
& \beta_{t}=\frac{\varepsilon_{t}}{1-\varepsilon_{t}} .
\end{aligned}
$$

4) Combine $T$ optimal weak classifier into a strong classifier and its compound mode is shown as

$$
\begin{aligned}
& c(x)= \begin{cases}1, & \sum_{t=1}^{T} \alpha_{t} h_{t}(x) \geqslant \frac{1}{2} \sum_{t=1}^{T} \alpha_{t} \\
0, & \text { otherwise }\end{cases} \\
& \alpha_{t}=\log \frac{1}{\beta_{t}}=\log \frac{1-\varepsilon_{t}}{\varepsilon_{t}}=-\log \varepsilon_{t} .
\end{aligned}
$$

After training the strong classifier, possible face region can be extracted as the input image. If the human face is detected in the input image, the classifier will output true. Otherwise, the classifier will output false. Therefore, the human face can be detected by means of the Adaboost algorithm.

\subsection{Classifier fusion}

The classical Adaboost algorithm can perform very well when it is used to detect the frontal face in an image. But in the practical application, many factors such as rotation and tilt of human face will influence the result of face detection. Therefore, studying a method to detect multi-angel face is necessary. For multi-angle face detection based on Adaboost algorithm, the profile cascade classifier must be applied. The profile cascade classifier can be trained by a large number of profile face samples and non-face samples. This paper puts forward a new method of classifier fusion, which is called the overlap separation method to detect multi-angle face. Combined with a large number of 
non-face samples, frontal face samples and profile face samples are adopted to train two different cascade classifiers. Two different classifiers detect possible frontal human face regions, profile human face regions and output preliminary detection results including frontal cascade fusion results and profile cascade fusion results for further research. There are three patterns of overlapped regions in these detection results from different cascade classifiers. These three patterns, which are shown in Fig. 5, include two detached regions, two regions with a small overlapped part and two regions with a large overlapped part. An overlap threshold $T_{\text {overlap }}$ is used to separate the overlapped face regions. It is defined as the ratio of the overlapped area to the minimum area between these two face regions. Experiments indicate that the maximum of correct detection rate appears when $T_{\text {overlap }}$ is set to 0.8 . Pattern 1 in Fig. 5 shows that these two detected face regions are detached and the overlapped ratio is less than $T_{\text {overlap. The preliminary detection re- }}$ sults include these two regions. Pattern 2 in Fig. 4 shows that the ratio of the overlapped area in the detected face regions to the minimum area between two face regions is less than $T_{\text {overlap }}$, both two detected face regions will be chosen as the preliminary results. Therefore, there are two preliminary detection results in both Patterns 1 and 2 after comparing with the overlapped ratio. In Pattern 3, the ratio of the overlapped area to the minimum area between two face regions is greater than $T_{\text {overlap }}$. So the finally preliminary detection result is its overlapped regions. Therefore, the overlapped separation method can reduce the number of redundant faces detected by classifier effectively and add the amount of possible faces.

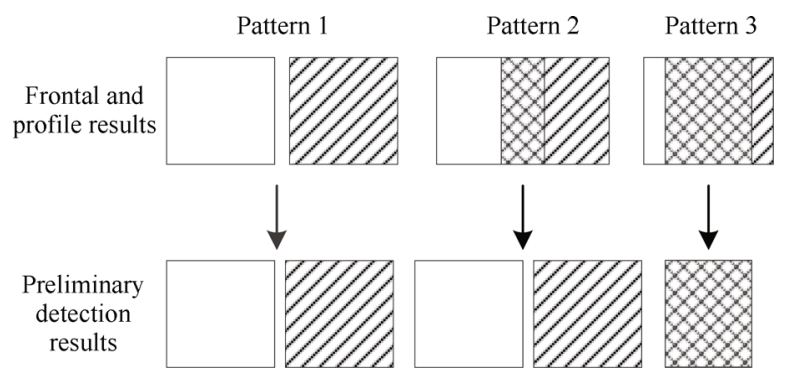

Fig. 5 Three cases of obtaining preliminary detection results

The process of classifier fusion with the overlap separation method is shown in Fig. 6. If there are the overlapped regions after applying frontal classifier and profile classifier, the ratio of the two overlapped areas in the detected face regions to the minimum area among these regions will be computed. Then by comparing the ratio value with $T_{\text {overlap }}$, the preliminary detection results can be obtained.

Fig. 7 gives an example photo with multi-angle faces to explain the effect of the classifier fusion with the overlapped separation method. When using the frontal classifier, two results can be obtained as shown in Fig. 7 (a). One of the two results is a woman face and the other is non-face image. The overlapped pattern of these two regions can be defined as Pattern 2 in Fig. 5. When using the profile classifier, one result can be obtained as shown in Fig. 7 (b). Therefore, by applying the overlapped separation method in Fig. 6, the preliminary detection results which include three detected face regions are shown in Fig. 7 (c).

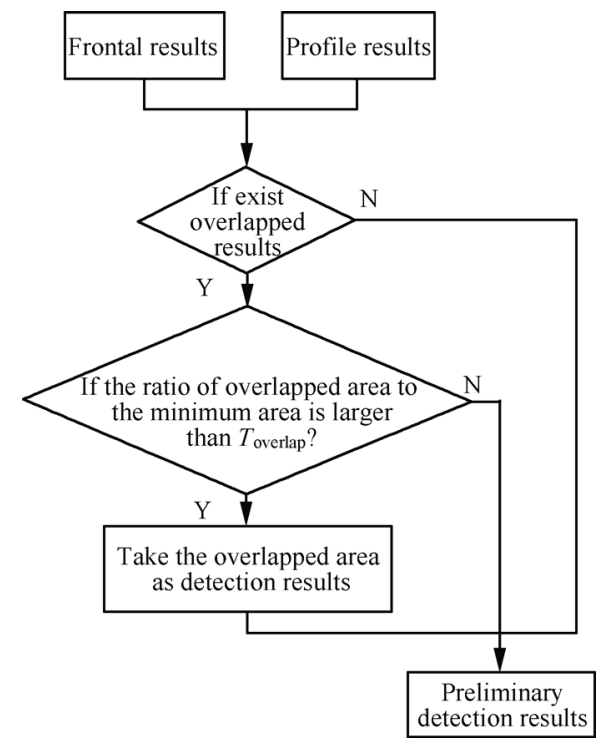

Fig. 6 The flow chart of classifier fusion with the overlap separation method

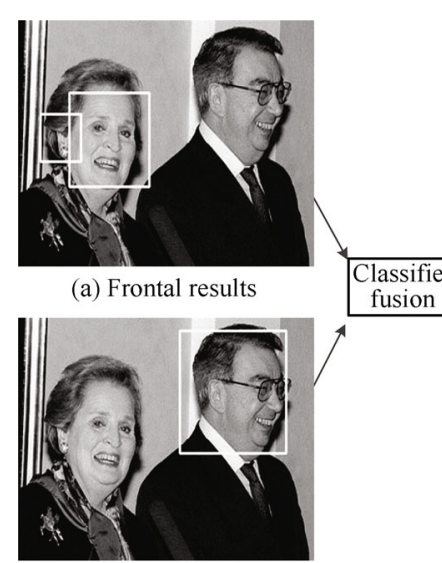

(b) Prontal results

Fig. 7 An example of multi-angle face detection by means of classifier fusion

\section{Remove false detection}

Eye is the window of soul, through which various psychological activities of the human can be explored. Because eyes are more obvious characteristics on human face than nose and mouth, face detection can be carried out by detecting the existence of these two eyes. If these two eyes exist in one image, this image may be considered as human face. Therefore, eye detection is a very important topic in automatic face analysis. It is used for preprocessing of face analysis $^{[21]}$. 
After applying the textual classifier fusion method to obtain preliminary detection results, many false faces may be found among these results, such as the preliminary results in Fig. 7 (c). An improved horizontal differential projection method is proposed in this paper to determine whether the region in the detection results is human face by means of analyzing if there are human eyes in the region or not. By applying this method, non-face parts in the preliminary results can be eliminated effectively.

There are two distinct features in eyes. One feature is that the color of eyes' white sclera is different from the other areas on human face. The other one is that the color of iris and pupil in eye region is darker than surrounding region on human face. Therefore, these gray levels in the eye region change drastically. According to the horizontal differential projection algorithm ${ }^{[22]}$, the position of eyes in an image with a frontal human face can be obviously found at the position of peak, which is shown in Fig. 8 (a). Therefore, it is effective to use classical horizontal differential projection algorithm to obtain these eye characteristics to determine whether frontal human faces exist in an image.

However, due to the complexity of practical face image, it is difficult to apply the classical horizontal differential projection algorithm directly to determine whether eyes exist in a preliminary detection regions. More than one peak may

appear in the result of the horizontal differential projection algorithm for the preliminary detection regions with profile faces and tilting faces. For example, for the tilting faces (Figs. $8(\mathrm{c})$ and $8(\mathrm{e})$ ) in which the line of eyes has some angles with respect to the horizontal line, two peak values may be usually obtained in the result of horizontal differential projection, such as the peaks in Figs. $8(\mathrm{~d})$ and $8(\mathrm{f})$.

An improved method of horizontal differential projection is put forward in this paper to find eyes in detection images no matter the line of eyes are horizontal or not. The implementation process of the algorithm to find if eyes exist in preliminary results is described as follows.

Step 1. Calculate these values of horizontal differential projection.

According to the prior knowledge of human face, two eyes always lie in the top two-thirds area of human face image, which is shown in Fig. 8 (a). In a preliminary detection region, these values of horizontal differential projection can be calculated by (10), where $D_{h(y)}$ is these values of horizontal differential projection and $f(x, y)$ is the pixel value at the coordinate point $(x, y)$. The range of $x$ is from $x_{1}$ to $x_{2}$, where $x_{1}$ is the left side of the image and $x_{2}$ is the right side of the image.

$$
D_{h}(y)=\sum_{x=x_{1}}^{x_{2}}|f(x+1, y)-f(x, y)| .
$$

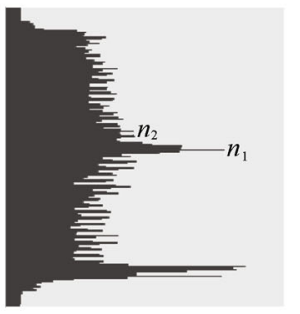

(b) The classical horizontal differentlal project of (a)

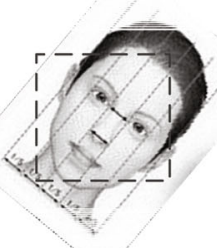

(e) Leaning to the right for 30 degrees

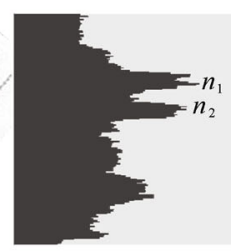

(f) The classical horizontal differential project of (d)

$$
\begin{array}{ll}
\text { (c) Leaning to } & \text { (d) The classical } \\
\text { the left for } 30 & \text { horizontal differen }
\end{array}
$$
degrees project of $(\mathrm{b})$

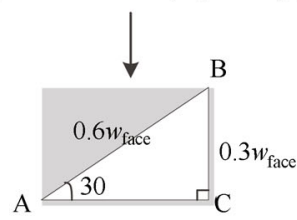

(g) Eyes position in the face leaning to left for 30 degrees

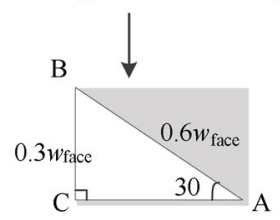

(h) Eye position in the face leaning to right for 30 degrees

Fig. 8 Standard face and its horizontal differential projection 
Step 2. Find the highest peak value $D_{h}\left(n_{1}\right)$.

Among these values of horizontal differential projection from (10), many peak values may be found, which are shown in Fig. 8 (d). The highest peak value must be found firstly for the further analysis. The highest peak value in the top two-thirds region of a human face image is defined as $D_{h}\left(n_{1}\right)$, where $n_{1}$ is the vertical position of the peak point. For example, in Figs. $8(\mathrm{~d})$ and $8(\mathrm{f})$, the values of $n_{1}$ are the highest peak values in these two images.

Step 3. Calculate the width of human face $W_{\text {face }}$.

These correctly preliminary detection results from Adaboost algorithm always contain a complete human face. Considering that the angle of tilting faces that are discussed in this paper is less than 30 degrees, the width of a preliminary detection image is approximately equal to the width of human face $W_{\text {face }}$.

Step 4. Calculate the position range of the second highest peak.

Considering the complexity of human face image, human eyes will generate more than one peak in the result, such as Figs. $8(\mathrm{~d})$ and 8 (f). Typically, the width of human eye is equal to $0.2 \times W_{\text {face }}$. If the tilting angle of human face in image is less than 30 degrees, the vertical length of one eye's projection in the result of horizontal different projection is $0.1 \times W_{\text {face }}$. Therefore, the peaks within the range from $n_{1}-0.05 \times W_{\text {face }}$ to $n_{1}+0.05 \times W_{\text {face }}$ will not be calculated because they may be generated by the eye which produces the highest peak $D_{h}\left(n_{1}\right)$. In Figs. $8(\mathrm{~g})$ and $8(\mathrm{~h})$, points $A$ and $B$ are defined as the distance between two eyes, which is approximately $0.6 \times W_{\text {face }}$. According to Fig. 8 (c), because the width of one eye is $0.2 \times W_{\text {face }}$, the distance between two eyes is $0.2 \times W_{\text {face }}$. Thus, if the tilting angle of human face in an image is less than 30 degrees, the maximum distance between points $A$ and $B$ in the horizontal projection result is about $0.3 \times W_{\text {face }}$. Finally, the position range of the second highest peak which is produced by the other eye must be in the top two-thirds area of human face image and be larger than $n_{1} \pm 0.05 \times W_{\text {face }}$ and be less than $n_{1} \pm 0.3 \times W_{\text {face }}$. For example, $n_{2}$ in Figs. 8 (d) and 8 (f) show the second highest peak value in the horizontal projection result.

Step 5. Find a position of the second eye.

If there is a second highest peak within the range that is discussed in Step 4, the position of this peak is defined as $n_{2}$ and the peak value is defined as $D_{h}\left(n_{2}\right)$. If (11) holds,

$$
\delta_{\text {eye }}=\frac{D_{h}\left(n_{1}\right)-D_{h}\left(n_{2}\right)}{D_{h}\left(n_{1}\right)}<T_{\text {eye }}
$$

where $\delta_{\text {eye }}$ is the relative error between two peaks and $T_{\text {eye }}$ is the setting threshold to determine the second eye. It means that the second highest peak $D_{h}\left(n_{2}\right)$ is close to the highest peak $D_{h}\left(n_{1}\right)$ and these two peaks indicate the position of two eyes in the detected regions. Because according to the analysis in Step 4, the distance between two eyes on human face must be small. In this case, the algorithm goes to Step 6. If (11) doesn't hold, one of these two peaks doesn't show the position of eyes and the algorithm goes to Step 7 .

Step 6. Calculate the weighted peak value.

If the highest peak and the second highest peak are found and (11) holds, a weighted peak value $D_{w}$ is calculated to enhance the peak value which is generated by two eyes. $D_{w}$ is described as

$$
D_{w}=D_{h}\left(n_{1}\right)+k D_{h}\left(n_{2}\right)
$$

where $k$ is a positive number from 0 to 1 . By applying $D_{w}$, these values of the improved horizontal differential projection $D_{H}(y)$ can be obtained by (13), which enhances the differential values of these two eyes to determine if human eyes exist in an image. When $y$ is between the positions of two peaks, a weighted peak value $D_{w}$ replaced the original result of horizontal differential projection $D_{h}(y)$. When $y$ does not locate in the above region, the original value $D_{h}\left(n_{2}\right)$ is used.

$$
D_{H}(y)=\left\{\begin{array}{c}
D_{w}, n_{1} \leqslant y \leqslant n_{2}, n_{1} \leqslant n_{2} \quad \text { or } \\
n_{2} \leqslant y \leqslant n_{1}, n_{2} \leqslant n_{1} \\
D_{h}(y), y \leqslant n_{1}, y \geqslant n_{2}, n_{1} \leqslant n_{2} \quad \text { or } \\
y \leqslant n_{2}, y \geqslant n_{1}, n_{2} \leqslant n_{1} .
\end{array}\right.
$$

Step 7. Remove false detection results.

There are two cases which come from Steps 5 and 6 respectively when drawing conclusion of algorithm to remove false results.

Case 1.

If a single peak value $D_{h}\left(n_{1}\right)$ is obtained in Step 5 , it is necessary to find the third highest peak value $D_{h}\left(n_{3}\right)$ in the top two-thirds area of the detected region. If a human face exists in the preliminary detection results, the difference between $D_{h}\left(n_{1}\right)$ and $D_{h}\left(n_{3}\right)$ should be much larger because the gray levels change drastically only in the eye region. The range of $D_{h}\left(n_{3}\right)$ must be in the top two-thirds area of human face image and be larger than $n_{1}+0.05 \times W_{\text {face }}$ and be smaller than $n_{1}-0.05 \times W_{\text {face. }}$ If (14) holds,

$$
\delta_{\text {peak }}=\frac{D_{h}\left(n_{1}\right)-D_{h}\left(n_{3}\right)}{D_{h}\left(n_{1}\right)}>T_{\text {peak }}
$$

where $\delta_{\text {peak }}$ is the relative error between $D_{h}\left(n_{1}\right)$ and $D_{h}\left(n_{3}\right)$ and $T_{\text {peak }}$ is the setting threshold to determine eyes' ${ }^{\prime}$ positions, all other peaks is much less than the highest peak $D_{h}\left(n_{1}\right)$ and $n_{1}$ indicates the position of human eyes in the detected region. Therefore, the final result of the algorithm is that a human face exits in the preliminary detection result.

\section{Case 2.}

If the weighted peak value $D_{w}$ is obtained in Step 6 , it is also necessary to find the third peak value $D_{h}\left(n_{3}\right)$. In this case, $n_{3}$ must be in the top two-thirds area of the detected region and within the following range. Equations (15) and (16) show that the third peak value $D_{h}\left(n_{3}\right)$ should appear outside of the area which is defined between $n_{1}$ and $n_{2}$.

$$
\begin{aligned}
& \text { If } n_{1} \geqslant n_{2} \\
& n_{3} \geqslant n_{1}+0.05 W_{\text {face }} \text { and } n_{3} \leqslant n_{2}-0.05 W_{\text {face }}
\end{aligned}
$$

If $n_{2} \geqslant n_{1}$

$n_{3} \geqslant n_{2}+0.05 W_{\text {face }}$ and $n_{3} \leqslant n_{1}-0.05 W_{\text {face }}$ 
If (17) holds,

$$
\delta_{\text {peak }}=\frac{D_{w}-D_{h}\left(n_{3}\right)}{D_{w}>T_{\text {peak }}}
$$

where $\delta_{\text {peak }}$ is the relative error between $D_{w}$ and $D_{h}\left(n_{3}\right)$, and $T_{\text {peak }}$ is the setting threshold to determine eyes' position, all other peaks are much less than the weighted peak $D_{w}$ and the range between $n_{1}$ and $n_{2}$ indicates the position of human eyes in the detected region. Therefore, the result of algorithm is that a human face exists in the preliminary detection results.

In the above two cases, if (14) or (17) does not hold, which means that the highest peak may be not the position of eyes, a conclusion can be drawn that there is no human face in the detected region. Finally, these false face regions among the preliminary detection results can be removed successfully.

The textual improved horizontal differential projection algorithm can be used to remove non-face results among three preliminary detection results from Fig. 7 (c). When using the textual algorithm, $k$ equals to $0.3, T_{\text {eye }}$ equals to 0.2 and $T_{\text {peak }}$ equals to 0.4 . Fig. 9 shows the results of the calculation.

In the horizontal differential projection of the first preliminary results (Fig. $9(\mathrm{~d})$ ), two peaks $n_{1}$ and $n_{2}$ can be found and a weighted peak value $D_{w}$ is calculated by (12). In Fig. $9(\mathrm{~g})$, the third peak $n_{3}$ can be found. However, the third peak $D_{h}\left(n_{3}\right)$ does not satisfy (17), which means that the relative error between $D_{w}$ and $D_{h}\left(n_{3}\right)$ is less than the threshold $T_{\text {peak }}$ and the position of $D_{w}$ may be not the position of eyes. Therefore, the conclusion is that the first preliminary region does not contain a human face. In the horizontal differential projection of the second preliminary region and the third preliminary region, peaks $n_{1}$ and $n_{2}$ can be also found in Figs. 9 (b) and (c). These two weighted peak values $D_{w}$ in Figs. $9(\mathrm{~h})$ and (i) can be calculated by (12), respectively. According to Step 6, the values of horizontal differential projection of eye region can be enhanced and the probability of misjudgment which is caused by the complexity of image is reduced. After calculation, the relative error between $D_{w}$ and $D_{h}\left(n_{3}\right)$ is more than the threshold $T_{\text {peak }}$, and the third peaks $D_{h}\left(n_{3}\right)$ in Figs. $9(\mathrm{~h})$ and (i) both satisfy (17). It means that the position of $D_{w}$ must be the position of human eyes. Therefore, the conclusion is that both the second preliminary region and the third preliminary region contain a human face. From this example, the textual algorithm can effectively judge whether these two eyes exist or not in the detected region by means of comparing the relative error between the highest peak $D_{h}\left(n_{1}\right)$ or $D_{w}$ and the third peak $D_{h}\left(n_{3}\right)$. So the false faces in the preliminary detection results can be found and removed even if human face tilts or human face rotates in the detected regions.

\section{Experimental results and discussion}

According to Sections 3 and 4, the textual DP-Adaboost algorithm with classifier fusion and improved horizontal differential projection method is programmed with $\mathrm{VC}++$ in VS2010 IDE. The training samples for frontal and profile human faces generate frontal cascade classifier and profile cascade classifier come from MIT face library and FERET face library. The size of each training sample is resized to the size of $20 \times 20$ pixels. All the train programs and experiments run in a computer with $2.20 \mathrm{GHz}$ Intel Core 2 Duo CPU and 2.00 GB RAM memory.

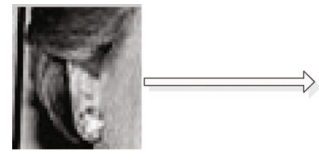

(a) The first preliminary result

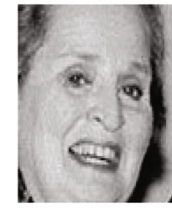

(b) The second preliminary result

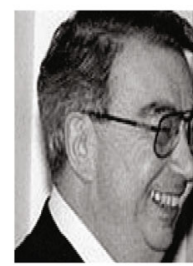

(c) The third possible face

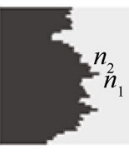

(d) Horizontal differentia projection of (a)

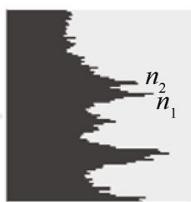

(e) Horizontal differential projection of (b)

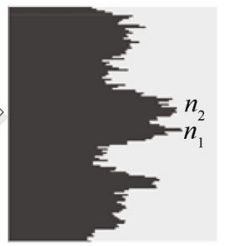

(f) Horizontal differential projection of (c)

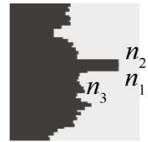

g) Improved horizontal differential projection of (a)

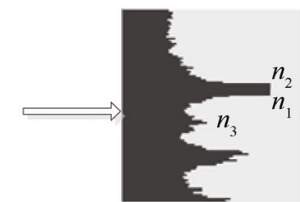

(h) Improved horizontal differential projection of (b)

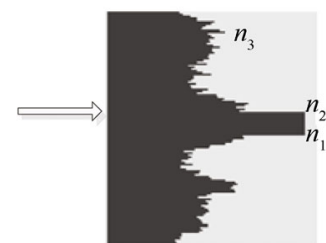

(i) Improved horizontal differential projection of (c)

Fig. 9 Examples of the improved horizontal differential projection method to judge human face in the preliminary detection results 
This paper adopts Gerogia Tech face database and CMU profile face test set to test the effect of the textual method for multi-angle face detection. The Gerogia Tech face database contains images of 50 people and each people has 15 color images with tilted face and cluttered background. The CMU profile face test set has 347 profile faces and 94 frontal faces.

Two typical test examples from the Gerogia Tech face database are shown in Figs. 10 and 11 to describe the textual method in detail. The face in Fig. 10 rotates to left by an angle of proximately 30 degrees. These two eyes are almost on the same horizontal line and stare straight ahead. The face in Fig. 11 tilts by about 30 degrees. These two eyes are not on the same horizontal line and look at the top.

Fig. 10 (a) shows 2 preliminary detection results in the first example with a rotating face. One is a rotating human face image which is marked with the big rectangle and the other is a false image which is marked with the small rectangle. These two preliminary detection results are obtained by the improved Adaboost algorithm with classifier fusion. Then, the improved horizontal differential projection algorithm is implemented to calculate the two preliminary results which are shown in Figs. 10 (b) and (e). Fig. 10 (c) gives the horizontal differential projection of a non-face image. Although it is not the human face, the highest peak $D_{h}\left(n_{1}\right)$ can be found in the top two-thirds area of the images and the second highest peak $D_{h}\left(n_{2}\right)$ within the range from $n_{1}-0.05 \times W_{\text {face }}$ to $n_{1}-0.3 \times W_{\text {face }}$ can be also found. Setting $T_{\text {eye }}$ equals to $0.2,(11)$ holds and the improved horizontal differential projection can be obtained and is shown in Fig. $10(\mathrm{~d})$. According to (16), $D_{h}\left(n_{3}\right)$ is found in Fig. $10(\mathrm{~d})$. Considering that $T_{\text {peak }}$ is set to 0.4, (17) cannot hold. This indicates that these two highest peaks in Fig. 10 (d) are not obvious enough to confirm the exist of eyes. Therefore, according to the textual algorithm, Fig. $10(\mathrm{~b})$ is a false detection image.

Fig. 10 (f) shows the horizontal differential projection of the second preliminary results in Fig. $10(\mathrm{e})$. The highest peak $D_{h}\left(n_{1}\right)$ in the top two-thirds area of the image and the second highest peak $D_{h}\left(n_{2}\right)$ within the range from $n_{1}+0.05 \times W_{\text {face }}$ to $n_{1}+0.3 \times W_{\text {face }}$ can be found in Fig. 10 (f). After calculation, it is found that (11) holds. By setting $k$ to 0.3 , the improved horizontal differential projection in Fig. 10 (g) can be obtained when applying (12), and $D_{h}\left(n_{3}\right)$ is found in Fig. $10(\mathrm{~g})$, which is much smaller than $D_{w}$. Therefore, (17) holds and Fig. 10 (e) is considered as a region with human face. The positions of two obvious peaks $n_{1}$ and $n_{2}$ in Fig. 10 (f) indicate the possible position of these two eyes. Because two eyes are on the same horizontal line in Fig. $10(\mathrm{e}), n_{1}$ is on behalf of the position of these two eyes. By comparing Figs. 10 (f) with 10 (e), another peak $n_{2}$ may be on behalf $\mathrm{f}$ the position of eyebrows.

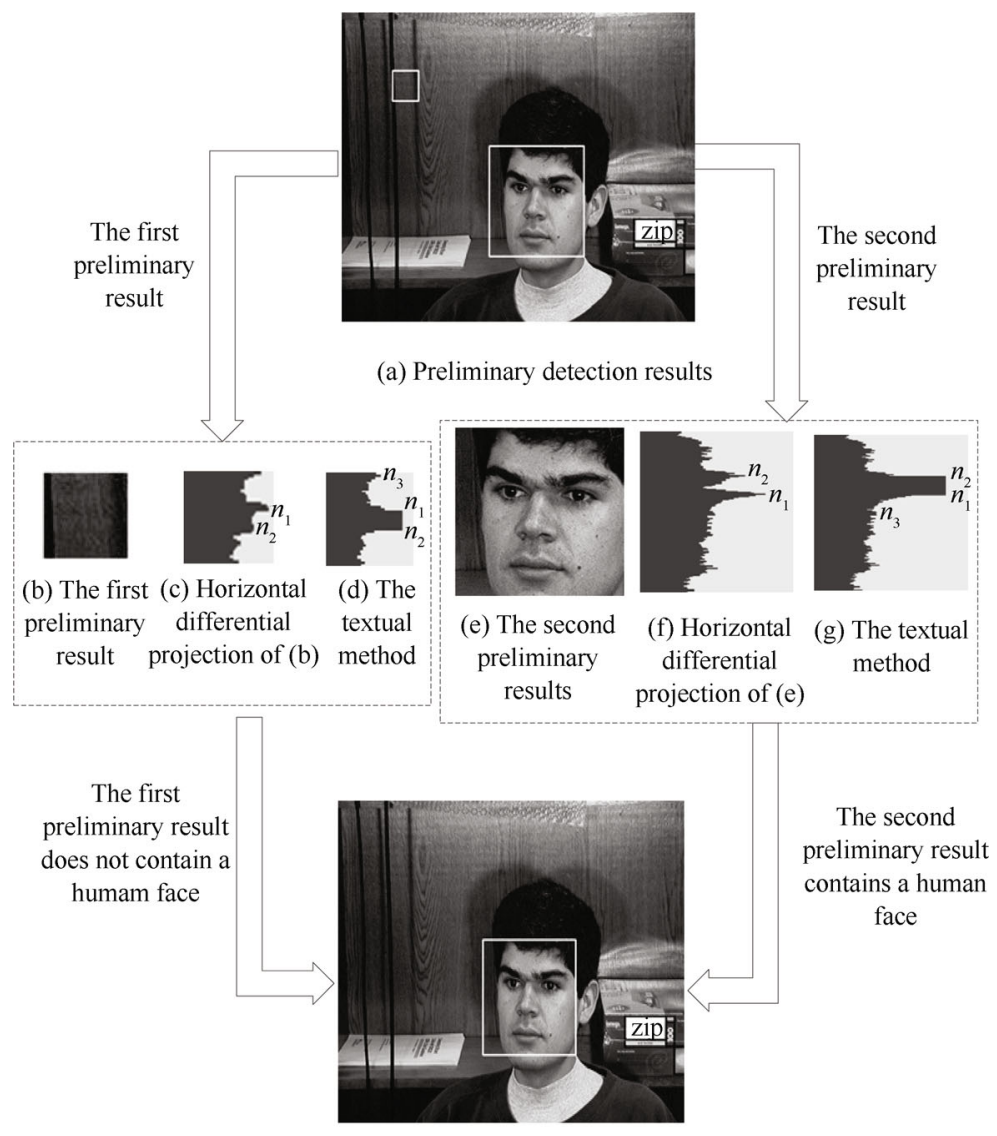

(h) The final result

Fig. 10 A typical example which is calculated with the textual DP-Adaboost algorithm 
Due to the dark eyebrows, differential calculation on them may result in higher peak value like $D_{h}\left(n_{2}\right)$ in Fig. 10 (f), which is usually a bit lower than the peak value $D_{h}\left(n_{1}\right)$ for eyes. Therefore, when using the textual algorithm, the regions of eyes and eyebrows may be recognized as eye region together.

All detailed data of the above calculation can be found in Table 1. Finally, Fig. 10 (h) shows that the correct detection result in the tested image is a human face marked with the big rectangle.

The textual algorithm is also implemented on the second example, and these three parameters $k, T_{\text {peak }}$ and $T_{\text {eye }}$ are set in accordance with the first example. In Fig. 11 (a), these two preliminary results, namely one tilting human face image which is marked with the big rectangle and one false image which is marked with the small rectangle, can be obtained by the improved Adaboost algorithm with classifier fusion. Fig. 11 (c) shows the horizontal differential projection of non-face image in Fig. 11(b). The highest peak $D_{h}\left(n_{1}\right)$ and the second highest peak $D_{h}\left(n_{2}\right)$ can be found in Fig. 11 (c). After calculation, (11) holds and the improved horizontal differential projection can be shown in Fig. $11(\mathrm{~d})$. According to (15), $D_{h}\left(n_{3}\right)$ is found in Fig. 11 (d) and (17) cannot hold. Therefore, Fig. 11 (b) is a false detection image.

Fig. 11 (f) shows the horizontal differential projection of Fig. $11(\mathrm{e})$. The positions of these two obvious peaks in Fig. 11 (f) are the positions of two eyes because of the tilting human face in Fig. 11 (e) tilts. Fig. 11 (g) can be obtained when applying (12). According to (15), $D_{h}\left(n_{3}\right)$ is found in Fig. $11(\mathrm{~g})$. After calculating, (17) holds and Fig. $11(\mathrm{e})$ is considered as a human face image. Comparing Fig. 11 (e) with Fig. 11 (f), because the line of two eyes on the human face has an obviously angle with the horizontal line due to the tilting face, these two obvious peaks, of which these values are closing, indicate the positions of two eyes.

Table 1 Detailed data after processing Fig. 10 by the textual algorithm

\begin{tabular}{ccc}
\hline Data & Non-face image & Human face image \\
\hline$W_{\text {face }}$ & 42 & 166 \\
$n_{1}$ & 18 & 65 \\
$D_{h}\left(n_{1}\right)$ & 1381 & 2029 \\
Range of $n_{2}$ & {$[6,15],[21,28]$} & {$[15,56],[74,109]$} \\
$n_{2}$ & 26 & 46 \\
$D_{h}\left(n_{2}\right)$ & 1138 & 1695 \\
$\delta_{\text {eye }}$ & 0.176 & 0.164 \\
$D_{w}$ & 1722 & 2538 \\
Range of $n_{3}$ & {$[1,15]$} & {$[1,37],[74,109]$} \\
$n_{3}$ & 2 & 75 \\
$D_{h}\left(n_{3}\right)$ & 1253 & 1239 \\
$\delta_{\text {peak }}$ & 0.272 & 0.512 \\
Conclusion & False & True \\
\hline
\end{tabular}

All detailed data of the above calculation can be found in Table 2. Finally, Fig. 11 (h) shows that the correct detection results are the image of a human face marked with the big rectangle.

Table 2 Detailed data after processing Fig. 11 by the textual algorithm

\begin{tabular}{ccc}
\hline Data & Non-face image & Human face image \\
\hline$W_{\text {face }}$ & 50 & 154 \\
$n_{1}$ & 2 & 73 \\
$D_{h}\left(n_{1}\right)$ & 537 & 1019 \\
Range of $n_{2}$ & {$[4,17]$} & {$[27,66],[80,102]$} \\
$n_{2}$ & 16 & 56 \\
$D_{h}\left(n_{2}\right)$ & 508 & 986 \\
$\delta_{\text {eye }}$ & 0.054 & 0.032 \\
$D_{w}$ & 706 & 1348 \\
Range of $n_{3}$ & {$[18,33]$} & {$[1,49],[80,102]$} \\
$n_{3}$ & 33 & 80 \\
$D_{h}\left(n_{3}\right)$ & 529 & 683 \\
$\delta_{\text {peak }}$ & 0.25 & 0.49 \\
Conclusion & False & True \\
\hline
\end{tabular}

These two typical examples in Figs. 10 and 11 have different face poses in the image. Fig. 10 has a rotating face and Fig. 11 has a tilting face. These two examples explain how the textual DP-Adaboost algorithm can successfully recognize human face with different poses in an image such as the frontal face, the rotating face and the tilting face, even if non-face regions are obtained from the Adaboost algorithm with classifier fusion.

Table 3 shows the comparative detection results between a single frontal classifier and the textual DP-Adaboost algorithm when all the images in Gerogia Tech face database and CMU profile face test set were tested. The results show that the textual method reduces the error rate from $11.92 \%$ to $5.54 \%$. Meantime, the improved horizontal differential projection algorithm can remove non-face region in the preliminary detection results. Due to the improved Adaboost algorithm with classifier fusion, many profile faces can be detected and the hit rate for face detection also can be improved from $68.35 \%$ to $71.87 \%$.

\section{Conclusions}

This paper proposes a new method called DP-Adaboost algorithm to detect multi-angle human face, which includes the improved Adaboost algorithm with classifier fusion for multi-angle face detection and the improved horizontal differential projection algorithm to remove false detection results. Experiment results show that compared with the classical Adaboost algorithm only with frontal classifier, the textual DP-Adaboost algorithm performs much better than in multi-angle face detection.

Table 3 The comparative detection results

\begin{tabular}{cccccc}
\hline Detection method & Face number & False alarms & False rate & Hits & Hit rate \\
\hline A single classifier & 1191 & 142 & $11.92 \%$ & 814 & $68.35 \%$ \\
DP-Adaboost & 1191 & 66 & $5.54 \%$ & 856 & $71.87 \%$ \\
\hline
\end{tabular}




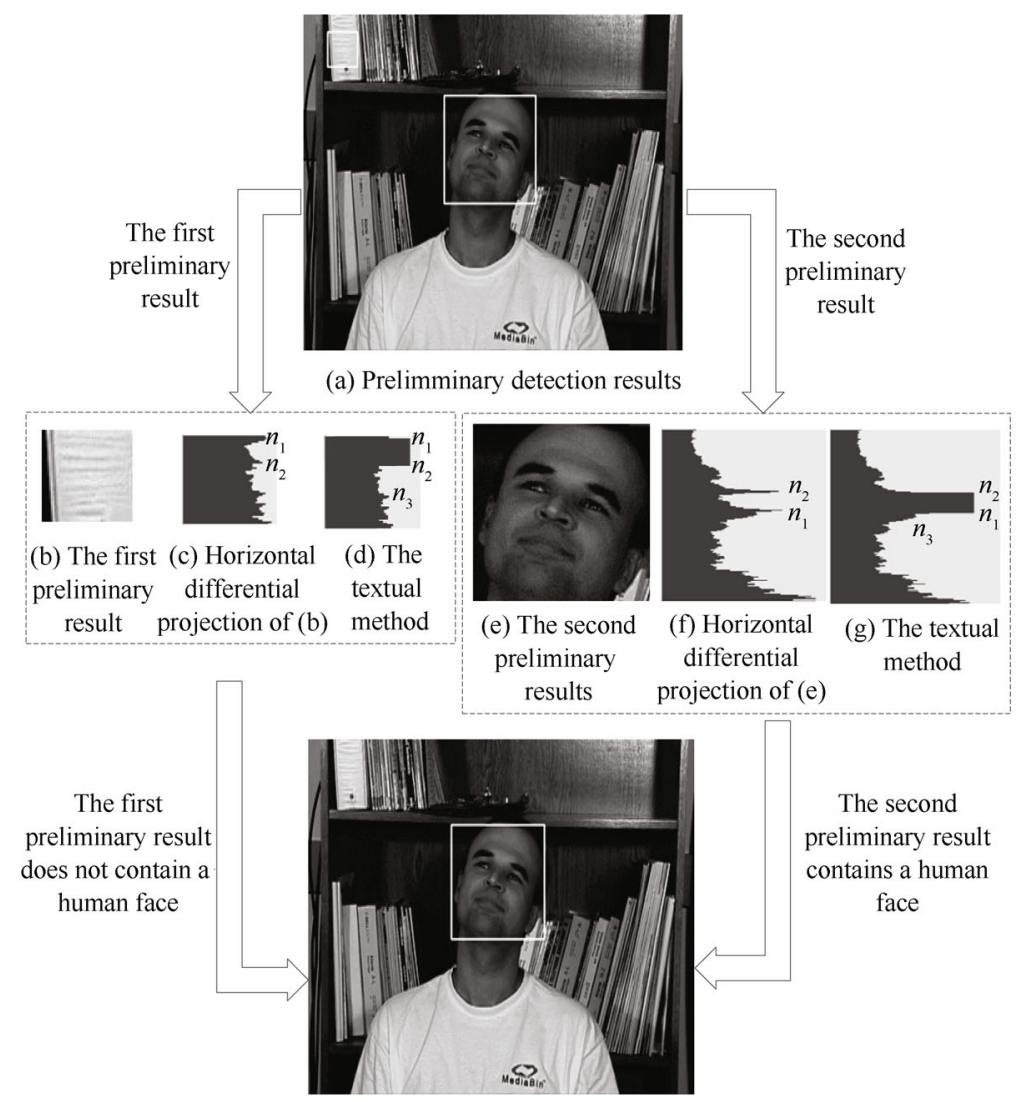

(h) The final result

Fig. 11 A typical example which is calculated with the textual DP-Adaboost algorithm

\section{References}

[1] B. J. Jun, D. J. Kim. Robust face detection using local gradient patterns and evidence accumulation. Pattern Recognition, vol. 45, no. 9, pp. 3304-3316, 2012.

[2] J. Satake, M. Chiba, J. Miura. Visual person identification using a distance-dependent appearance model for a person following robot. International Journal of Automation and Computing, vol. 10, no. 5, pp. 438-446, 2013.

[3] A. A. Shafie, A. B. M. Ibrahim, M. M. Rashid. Smart objects identification system for robotic surveillance. International Journal of Automation and Computing, vol. 11, no. 1, pp. 59-71, 2014.

[4] M. Turk, A. Pentland. Eigenfaces for recognition. Cognitive Neuroscience, vol. 3, no. 1, pp. 71-86, 1991.

[5] G. Yang, T. S. Hang. Human face detection in a complex background. Pattern Recognition, vol. 27, no. 1, pp. 53-63, 1994.

[6] T. F. Cootes, C. J. Taylor, D. H. Cooper. Active shape models - their training and application. Computer Vision and Image Understanding, vol.61, no. 1, pp. 38-59, 1995.

[7] E. Osuna, R. Freund, F. Girosi. Training support vector machines: An application to face detection. In Proceedings of IEEE Computer Society Conference on Computer Vision and Pattern Recognition, IEEE, San Juan, USA, pp. 130$136,1997$.
[8] P. Viola, M. Jones. Rapid object detection using a boosted cascade of simple features. In Proceedings of IEEE Computer Society Conference on Computer Vision and Pattern Recognition, IEEE, Kauai, USA, pp. 1511-1518, 2001.

[9] Y. W. Wang. Multi-view Face Detection Based on Skin Color and Adaboost Algorithm, Master dissertation, Beijing Jiaotong University, China, 2011. (in Chinese)

[10] Y. Z. Lu. A novel face recognition algorithm for distinguishing faces with various angles. International Journal of $\mathrm{Au}$ tomation and Computing, vol. 5, no. 2, pp. 193-197, 2008.

[11] K. H. B. Ghazali, J. Ma, R. Xiao. Multi-angle face detection using back propagation neural network. In Proceedings of the 2nd International Conference on Frontiers of Manufacturing and Design Science, Taichung, Taiwan, China vol. 121-126, pp. 2411-2415, 2012.

[12] S. Y. Yu. Study of Multi-view Face Recognition Based on Active Appearance Model, Master dissertation, Zhejiang Normal University, China, 2011. (in Chinese)

[13] H. A. Rowley, S. Baluja, T. Kanade. Rotation invariant neural network-based face detection. In Proceedings of IEEE Computer Society Conference on Computer Vision and Pattern Recognition, IEEE, Santa Barbara, USA, pp. 38-44, 1998.

[14] S. Z. Li, Z. Q. Zhang. FloatBoost learning and statistical face detection. IEEE Transactions on Pattern Analysis and Machine Intelligence, vol. 26, no. 9, pp. 1112-1123, 2004. 
[15] H. Schneiderman, T. Kanade. A statistical method for 3D object detection applied to faces and cars. In Proceedings of IEEE Conference on Computer Vision and Pattern Recognition, Hilton Head Island, USA, vol. 1, pp. 746-751, 2000.

[16] X. L. Zong, S. W. Xiong, G. F. Zhu. The detection of colorful face image based on skin color and Adaboost algorithm. Application Research of Computers, vol. 24, no. 10, pp. 178$180,2007$.

[17] R. Lienhart, J. Maydt. An extended set of Haar-like features for rapid object detection. In Proceedings of International Conference on Image Processing, IEEE, Rochester, USA, vol. 1, pp. 900-903, 2002.

[18] C. C. Hsieh, D. H. Liou. Novel Haar features for real-time hand gesture recognition using SVM. Journal of Real-time Image Processing, vol. 10, no. 2, pp. 357-370, 2012.

[19] W. J. Liao. Research and Implementation on Multi-angle Face Detection Technology Based on Continuous Adaboost Algorithm, Master dissertation, Nanjing University of Posts and Telecommunications, China, 2012. (in Chinese)

[20] G. Li, Y. P. Xu, J. Y. Wang. An improved AdaBoost face detection algorithm based on optimizing skin color model. In Proceedings of the 6th International Conference on Natural Computation, IEEE, Yantai, China, vol.4, pp. 20132015,2010

[21] P. Campadelli, R. Lanzarotti, G. Lipori. Precise eye and mouth localization. International Journal of Pattern Recognition, vol. 23, no. 3, pp. 359-377, 2009.
[22] J. Y. Cao, Y. L. Zhao. An algorithm to locate the eyes in driver's face recognition. Journal of Xiangnan University, vol. 28, no. 5, pp. 76-79, 2007. (in Chinese)

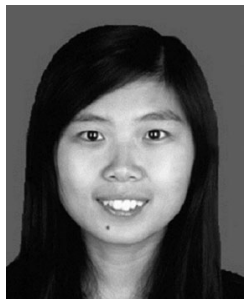

Ying-Ying Zheng is a master student in mechanical \& electronic engineering and automation of Shanghai University, China.

Her research interests include image processing and machine vision.

E-mail: cocoatang@126.com

ORCID iD: 0000-0002-9484-5113

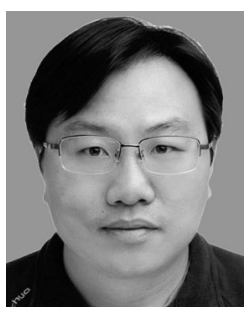

Jun Yao received the B.Sc. degrees in industrial automation from the Shanghai University, China in 2000, received the $\mathrm{Ph}$. D. degree in control theory and engineering from Shanghai University, China in 2006. Currently, he is a lecturer in the School of Mechanical \& Electronic Engineering and Automation, Shanghai University, China. He has published about 20 refereed journals, conference papers and patents.

His research interests include machine vision, digital image processing, and signal processing in detecting system.

E-mail: grandone0529@shu.edu.cn (Corresponding author) ORCID iD: 0000-0002-5438-077X 